\title{
Video Article \\ High-Throughput Total Internal Reflection Fluorescence and Direct Stochastic Optical Reconstruction Microscopy Using a Photonic Chip
}

\author{
David André Coucheron ${ }^{1}, \varnothing y s t e i n$ Ivar Helle ${ }^{1}$, Cristina Ionica $\varnothing i e^{2}$, Jean-Claude Tinguely ${ }^{1}$, Balpreet Singh Ahluwalia ${ }^{1}$ \\ ${ }^{1}$ Department of Physics and Technology, UiT The Arctic University of Norway \\ ${ }^{2}$ Vascular Biology Research Group, Department of Medical Biology, UiT The Arctic University of Norway
}

Correspondence to: Jean-Claude Tinguely at jean-claude.tinguely@uit.no

URL: https://www.jove.com/video/60378

DOI: doi:10.3791/60378

Keywords: Biochemistry, Issue 153, Fluorescence, total internal reflection fluorescence, Super-resolution optical microscopy, nanoscopy, waveguides, integrated optics

Date Published: $11 / 16 / 2019$

Citation: Coucheron, D.A., Helle, Ø.I., Øie, C.I., Tinguely, J.C., Ahluwalia, B.S. High-Throughput Total Internal Reflection Fluorescence and Direct Stochastic Optical Reconstruction Microscopy Using a Photonic Chip. J. Vis. Exp. (153), e60378, doi:10.3791/60378 (2019).

\section{Abstract}

Total internal reflection fluorescence (TIRF) is commonly used in single molecule localization based super-resolution microscopy as it gives enhanced contrast due to optical sectioning. The conventional approach is to use high numerical aperture microscope TIRF objectives for both excitation and collection, severely limiting the field of view and throughput. We present a novel approach to generating TIRF excitation for imaging with optical waveguides, called chip-based nanoscopy. The aim of this protocol is to demonstrate how chip-based imaging is performed in an already built setup. The main advantage of chip-based nanoscopy is that the excitation and collection pathways are decoupled. Imaging can then be done with a low magnification lens, resulting in large field of view TIRF images, at the price of a small reduction in resolution. Liver sinusoidal endothelial cells (LSECs) were imaged using direct stochastic optical reconstruction microscopy (dSTORM), showing a resolution comparable to traditional super-resolution microscopes. In addition, we demonstrate the high-throughput capabilities by imaging a $500 \mu \mathrm{m} \times 500$ $\mu \mathrm{m}$ region with a low magnification lens, providing a resolution of $76 \mathrm{~nm}$. Through its compact character, chip-based imaging can be retrofitted into most common microscopes and can be combined with other on-chip optical techniques, such as on-chip sensing, spectroscopy, optical trapping, etc. The technique is thus ideally suited for high throughput 2D super-resolution imaging, but also offers great opportunities for multimodal analysis.

\section{Video Link}

The video component of this article can be found at https://www.jove.com/video/60378/

\section{Introduction}

Since the initial demonstration of single molecule localization microscopy, many variations have been developed to solve different challenges ${ }^{1,2,3}$ One challenge that has remained, however, is large field of view dSTORM imaging. Many dSTORM setups use the same objective lens to both excite the sample and to image it. In order to increase the field of view, a low magnification lens is needed. Low magnification and low numerical aperture (NA) objective lenses typically have a large depth of field, resulting in an increased out-of-plane signal that will reduce the localization precision. TIRF objectives are commonly used to increase the image contrast by reducing out-of-plane fluorescence. Through TIRF, the excitation is limited to an optical thickness of approximately $150 \mathrm{~nm}$ from the surface by means of an evanescent field ${ }^{4}$. TIRF objective lenses require a large NA resulting in a small field-of-view (FOV) (e.g., $50 \times 50 \mu \mathrm{m}^{2}$ ), which limits the throughput significantly. There are, however, alternative ways to generate an evanescent field.

An optical waveguide is a structure that will confine and guide light if it is coupled into the structure. Most commonly, waveguides are used in fiber-based telecommunication. Great effort has been made in order to develop 2D integrated waveguides as a main component of photonic integrated circuits. The technology has advanced to a point where fabricating low-loss nano-structured optical waveguides can be routinely done ${ }^{5}$. Today, several foundries around the world can be used to develop photonic integrated circuits. Waveguides guide light through total internal reflectance also exhibiting an evanescent field at the surface. By careful design of the waveguide structure, a high intensity can be achieved in the evanescent field. A sample placed directly on top of the waveguide surface can thus also be illuminated by the evanescent field for imaging applications. The evanescent field will be generated along the entire length and width of the waveguide, and thus it can be made arbitrarily large 6 .

We present a novel approach to TIRF dSTORM that offers an arbitrarily large field of view. Instead of using a TIRF lens for both excitation and collection, we excite using the evanescent field from optical waveguides. This decouples the excitation and collection light pathway, allowing for total freedom along the collection light path without compromising the optical sectioning for a given wavelength provided by the waveguide chip illumination. Low magnification lenses can thus be used to image very large regions in TIRF mode, although a smaller NA will reduce the lateral resolution. Furthermore, multicolor imaging is also greatly simplified using waveguides ${ }^{7}$, as several wavelengths can be guided and detected without readjusting the system. This is advantageous for dSTORM, as low wavelengths can be used to enhance fluorophore blinking and for multicolor imaging. It is worth noting that the penetration depth of the evanescent field will change as a function of wavelength, although 
it does not affect how the imaging procedure is performed. The chip is compatible with live cell imaging ${ }^{8}$ and is ideal for applications such as the integration of microfluidics. Each chip can contain tens of waveguides, which can allow the user to image under different conditions or apply optical trapping ${ }^{9}$ and Raman spectroscopy ${ }^{10}$.

The chip-based system works equally well for both diffraction-limited and super-resolution imaging. A similar approach was introduced in 2005 using a prism to generate evanescent field excitation ${ }^{4}$. The photonic chip also excites through the evanescent field, but with modern waveguide fabrication techniques, one can generate exotic light patterns with waveguides. The present chip-based nanoscopy implementation is limited to $2 \mathrm{D}$ imaging only, as the excitation field is locked inside the waveguide surface. Future development will aim for 3D applications. Additionally, other super-resolution techniques such as structured illumination microscopy are being developed using the same chip-based microscope ${ }^{11}$.

\section{Protocol}

\section{Preparation of the polydimethylsiloxane (PDMS) layer}

1. Prepare a $10: 1$ mix of Sylgard 184 monomer and curing agent.

2. Place the mixture in a vacuum chamber until air bubbles are gone.

3. Pour $1.7 \mathrm{~g}$ of PDMS mixture in the center of a 3.5 inch (diameter) Petri dish.

4. Place the Petri dish on the vacuum chuck of a spin coater.

5. Spin coat the Petri dish for $20 \mathrm{~s}$ at $900 \mathrm{rpm}$, with an acceleration of $75 \mathrm{rpm} / \mathrm{s}$.

6. Cure the dish on a hotplate at $50{ }^{\circ} \mathrm{C}$ for at least $2 \mathrm{~h}$.

\section{Sample preparation}

1. Waveguide cleaning

1. Prepare $100 \mathrm{~mL}$ of a $1 \%$ dilution of cleaning detergent concentrate (Table of Materials) in deionized (DI) water.

2. Place the chip in a glass Petri dish using a wafer tweezer and cover completely with the detergent solution.

3. Place the Petri dish on a hot plate at $70{ }^{\circ} \mathrm{C}$ for $10 \mathrm{~min}$.

4. While still on the hot plate, rub the surface with a cleanroom tissue swab.

5. Remove the chip from the Petri dish. Rinse with at least $100 \mathrm{~mL}$ of DI water.

6. Rinse with at least $100 \mathrm{~mL}$ of isopropanol, taking care that solvent does not dry on the surface to prevent evaporation stains.

7. Rinse with at least $100 \mathrm{~mL}$ of DI water. Blow the chip dry with a nitrogen gun.

\section{Chamber preparation}

1. Prepare a layer of $150 \mu \mathrm{m}$ polydimethylsiloxane (PDMS) in a Petri dish (section 1).

2. Use a scalpel to cut a $1.5 \mathrm{~cm} \times 1.5 \mathrm{~cm}$ frame from the PDMS layer.

3. Lift the frame from the Petri dish with a tweezer. Deposit it flat on a clean and polished chip. The sample is now ready for cell seeding

\section{Fluorescent labeling}

1. Prepare the following chemicals: phosphate-buffered saline solution, dye solution(s), dSTORM imaging buffer.

2. After cell seeding, remove the chip from the media.

3. Use a pipette to remove any excess fluid from outside the PDMS chamber.

4. Remove the current fluid from inside the PDMS chamber with a pipette while adding approximately $60 \mu \mathrm{L}$ of clean PBS at the same time.

NOTE: The amount added to the chamber will have to be changed according to the chamber size. Be careful not to remove all media from the cell surface.

5. Replace the PBS with $60 \mu \mathrm{L}$ of clean PBS and let it incubate for $1 \mathrm{~min}$

6. Repeat the previous step, letting it incubate for $5 \mathrm{~min}$ this time.

7. Remove the PBS and replace it with $60 \mu \mathrm{L}$ of the dye solution. Leave the sample to incubate for around 15 minutes, shielding it from light.

NOTE: This step might have to change significantly, depending on the fluorescent dye used. We use CellMask Deep Red to label the cell membrane for this experiment

8. Wash the sample with PBS as in steps 2.3.3-2.3.5.

9. Remove the PBS and replace it with $40 \mu \mathrm{L}$ of the imaging buffer at the same time. NOTE: There are several different imaging buffers for different fluorescent dyes.

10. Place a coverslip on top, preventing air bubbles from forming underneath. Gently press the coverslip against the imaging chamber to remove any excess media.

11. Use a pipette to remove any excess media outside the coverslip. Clean the area outside the coverslip with a water moist swab to avoid crystals formed by dried immersion media residues.

\section{Imaging procedure}

1. Component setup

NOTE: This version of the setup consists of three main components: the microscope, coupling stage, and sample stage. See the Table of Materials.

1. Use a microscope with a filter holder, white light source, camera, and objective revolver.

2. Use a 3-axis piezo coupling stage with a fiber-coupled laser and a coupling lens.

3. Use a one-axis manual sample stage with tip and tilt and a vacuum holder. 
4. Mount both the coupling and the sample stage on a 2-axis motorized stage for sample translation.

\section{Waveguide coupling}

1. Place the chip on the vacuum chuck with the coupling facet towards the coupling objective. Make sure the chip is approximately one focal length away from the coupling objective.

2. Turn on the vacuum pump.

3. Turn on the laser to $1 \mathrm{~mW}$. Roughly adjust the chip height so that the beam hits the edge of it. Turn off the laser.

4. Turn on the white light source. Choose a low magnification objective lens (e.g. 10x). Focus the microscope on a waveguide.

5. Translate the microscope along the waveguide to see if it is well aligned with the optical path. Move the microscope to the coupling edge.

6. Turn on the laser at $1 \mathrm{~mW}$ or less. Translate the microscope along the coupling edge to find the laser light. Focus the beam on the chip edge.

7. Adjust the coupling stage along the optical path in the direction that reduces the laser beam spot size until it disappears. The beam is now either above or below the chip surface.

8. Adjust the coupling stage height until the beam spot reappears and is maximized.

9. Repeat the two previous steps until the laser forms a focused spot.

10. Move the focused spot to the waveguide of interest.

11. Translate the microscope a short distance away from the edge so that the focused beam spot is no longer visible. Turn off the white light.

12. Adjust the contrast. If the waveguide is guiding, the scattered light along the waveguide should be clearly visible.

13. Adjust the axes of the coupling stage to maximize the scattered light intensity. Turn off the laser.

14. Turn on the white light. Adjust the contrast if necessary.

15. Navigate to the imaging region.

3. Diffraction limited imaging

1. Focus with the desired imaging objective. Turn the white light off.

2. Insert the fluorescence filter and turn the laser power to $1 \mathrm{~mW}$.

3. Set the camera exposure time to approximately $100 \mathrm{~ms}$. Adjust the contrast as needed. Ensure that the coupling is still optimized.

4. Locate a region of interest for imaging. Turn on the piezo stage looping to average out modes.

NOTE: $20 \mu \mathrm{m}$ scan range with a step size of $50 \mathrm{~nm}$ is suitable for most waveguide structures.

5. Capture at least 300 images.

6. Load the captured image stack to Fiji using a virtual stack. From the image menu in Fiji, choose Stacks and z-project.

7. Calculate the TIRF image by choosing projection type average intensity.

4. dSTORM imaging

1. Turn on the laser to $1 \mathrm{~mW}$ and set the camera exposure time to $30 \mathrm{~ms}$.

2. Adjust the contrast and focus.

3. Increase the laser power until blinking is observed.

NOTE: This might take a while, depending on evanescent field intensity.

4. Zoom in on a small region of the sample.

5. Adjust the contrast.

6. Capture a few images to see if the blinks are well separated.

7. Adjust the camera exposure time for optimal blinking.

NOTE: Optimizing blinking is a complex task, but a lot of suitable literature is available ${ }^{12}$.

8. Turn on the piezo stage looping.

9. Record an image stack of at least 30,000 frames, depending on the blinking density.

\section{5. dSTORM image reconstruction}

1. Open Fiji and load the $d$ STORM stack as virtual images.

2. Adjust the contrast, if necessary.

3. Use the rectangle tool to select the area to reconstruct.

4. Open Run analysis in the Thunderstorm ${ }^{13}$ plugin in Fiji.

5. Set the basic camera settings in Thunderstorm corresponding to the device. The remaining default parameters are usually satisfactory.

6. Start the reconstruction.

NOTE: For the full field of view, the data might need to be divided in substacks, due to the large file size.

7. Filter the localization list provided by the reconstruction software to remove unspecific localizations. Apple an additional drift-correction if necessary.

\section{Representative Results}

TIRF microscopy is a popular technique as it removes out-of-plane fluorescence, increases contrast and thus improves image quality, and is less phototoxic compared to other fluorescence based microscopy techniques. Compared to the traditional objective-based approach, chip-based microscopy offers TIRF excitation without the limited throughput that is usually accompanied with a TIRF lens. An overview of the presented setup can be found in Figure 1A. We present diffraction-limited as well as dSTORM images of liver sinusoidal endothelial cells (LSEC) extracted from mice. A large field of view image of LSECs with labelled microtubulin is also presented, demonstrating the capabilities of high throughput imaging. A conventional dSTORM setup using an oil immersion TIRF lens (either $60 \mathrm{x}$ or $100 \mathrm{x}$ magnification) typically images an area of $50 \mu \mathrm{m} x$ $50 \mu \mathrm{m}$, which is 100 times smaller than the chip-based image in Figure 2, imaged with a 25x, 0.8 NA objective. 
In this method, we use multi-moded $\mathrm{Si}_{3} \mathrm{~N}_{4}$ waveguides for excitation. The utilized chips consist of a strip-etched guiding layer of $150 \mathrm{~nm} \mathrm{Si}{ }_{3} \mathrm{~N}_{4}$ deposited over a $2 \mu \mathrm{m}$ oxidized layer of a silicon chip. A schematic of the chip can be found in Figure 1B. Waveguide widths can vary between 200 and $1000 \mu \mathrm{m}$. Fabrication details can be found elsewhere ${ }^{8}$. Through interference between the propagating modes the excitation light will not have a homogeneous intensity distribution, but rather a spatially varying pattern. Figure $2 \mathrm{~A}$ presents an image with clearly visible mode patterns. This interference pattern will change with the position of the laser beam at the edge of the waveguide. In order to achieve homogeneous excitation in the final images, we use a piezo stage to oscillate along the coupled facet. Over the course of the imaging procedure, enough variation of the interference patterns exists so that they can be averaged, removing intensity fluctuations in the image. The image stack will consist of several images such as in Figure 2A, although with different patterns, but when averaged, the stack will yield an image with homogeneous excitation such as Figure 2B. An alternative approach is to use adiabatic tapering to achieve wide, single moded waveguides ${ }^{8,14}$, which removes the necessity of mode averaging. However, several millimeters of tapering length are necessary to maintain the single-mode condition to achieve a $100 \mu \mathrm{m}$ waveguide width. Multi-moded waveguides circumvent this tapering necessity and leave no limitations on the structure width. Beyond the illumination pattern, the highly effective refractive index of the modes allow for unprecedented possibilities towards structured illumination microscopy ${ }^{11}$ and fluctuation microscopy methods ${ }^{7}$.

The first step in imaging is to collect a diffraction limited image. The experiment results in a stack of around 300 images and the final image is made by taking the average of the stack. In Figure 2, we present diffraction limited and dSTORM imaging of LSECs labelled with CellMask Deep Red using a 60x, 1.2 NA water immersion objective. Figure 2A shows inhomogeneous illumination caused by insufficient mode averaging Successful mode averaging is displayed in Figure 2B. Figure $\mathbf{2 C}$ is a dSTORM image of the same region, with the marked region shown in Figure 2D. Liver sinusoidal endothelial cells have nano-sized pores in the plasma membrane ${ }^{15}$, which can be seen here. A Fourier Ring Correlation analysis provided a resolution of $46 \mathrm{~nm}$.

Figure 3 presents a dSTORM image of a $500 \mu \mathrm{m} \times 500 \mu \mathrm{m}$ region, demonstrating the high throughput capabilities of the technique. A zoomed image of Figure 3A, corresponding to a typical dSTORM field-of-view, is presented together with the diffraction limited image in Figure 3B. A Fourier ring correlation to estimate the resolution was performed, yielding a value of $76 \mathrm{~nm}$.
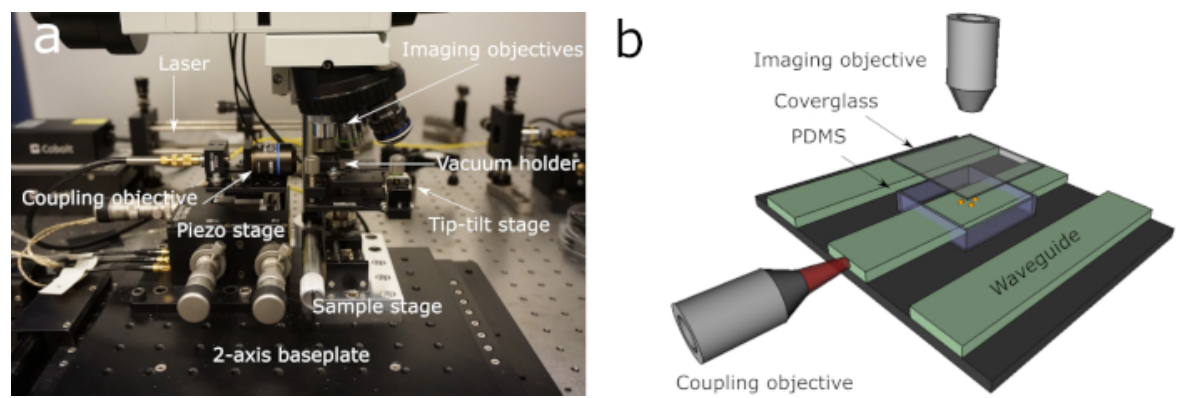

Figure 1: Imaging system and waveguide. (A) Photograph of the imaging system. The sample is placed on a vacuum chuck on the sample stage, with the coupling facet of the waveguide towards the coupling objective. A fiber coupled laser and a coupling objective is placed on top of a 3D piezo stage. A lens turret with imaging lenses captures the image from above and relays it to a camera. (B) Schematic of the waveguide with coupling and imaging lenses. The coupling lens couples light into the waveguide. The samples (orange beads) are kept inside a sealed PDMS chamber. The evanescent field along the waveguide will excite the sample and the imaging objective will capture the emitted fluorescence. Please click here to view a larger version of this figure. 

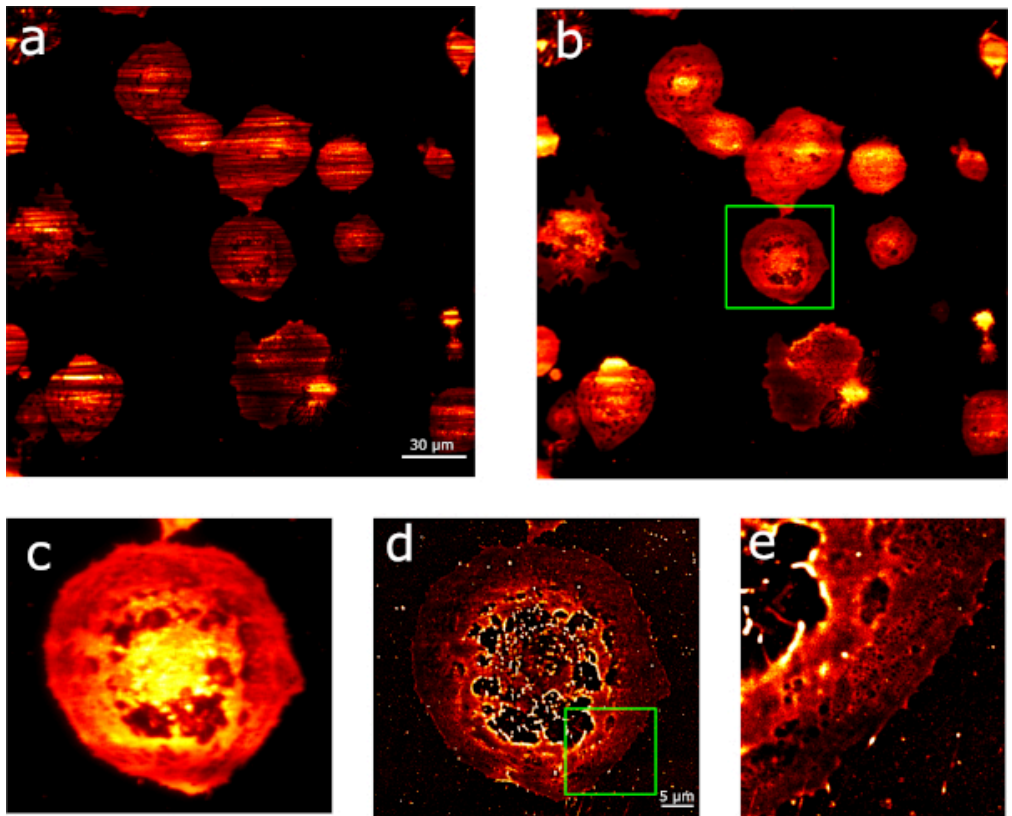

Figure 2: Diffraction-limited and dSTORM images. (A) Image of liver sinusoidal endothelial cells with insufficient mode averaging, resulting in a clearly visible excitation pattern. (B) The same region as in (A), but with sufficient mode averaging, resulting in homogeneous excitation. (C) Diffraction limited image of the inset in (B); (D) dSTORM image of the same region. (E) Inset of (D), clearly showing the fenestrations in the plasma membrane of the cell. Please click here to view a larger version of this figure.

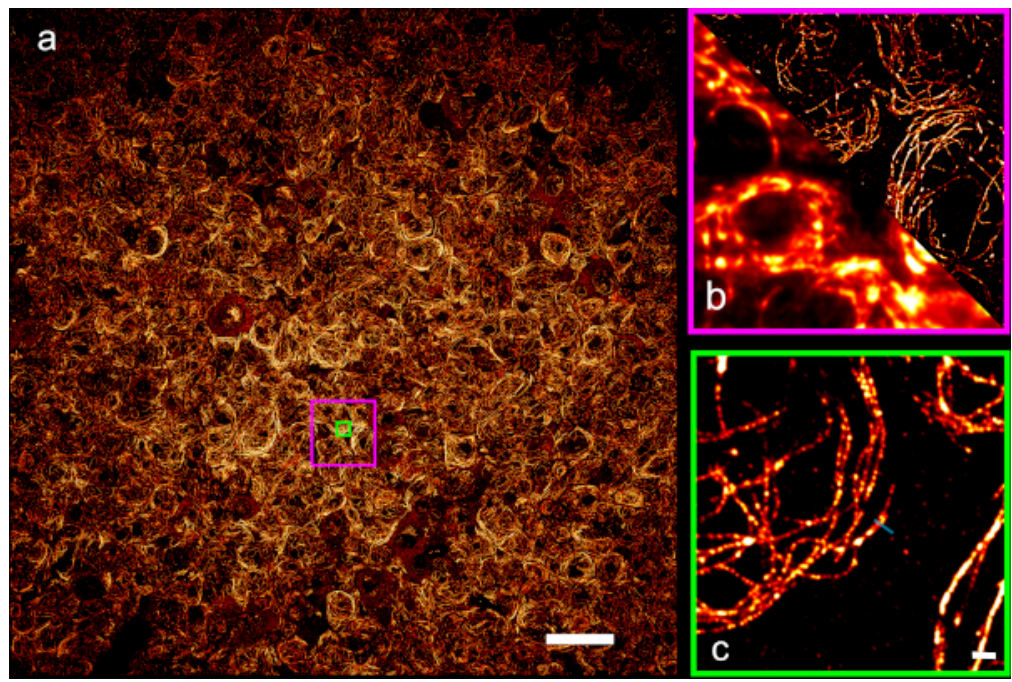

Figure 3: dSTORM image of rat LSECs. (A) Large field of view dSTORM image of Alexa 647 stained tubulin in rat LSECs. Scale bar $=50 \mu m$. (B) Larger marked region from (A) comparing diffraction-limited (bottom left) and dSTORM image (top right). (C) Smaller marked region from (A). Scale bar $=1 \mu \mathrm{m}$. The image has a resolution of $76 \mathrm{~nm}$. Adapted with permission from Helle et al. $2019^{6}$. Please click here to view a larger version of this figure.

\section{Discussion}

Chip-based imaging is similar to conventional dSTORM imaging. Image quality can thus be gauged using the same approaches as for traditional dSTORM imaging. The main difference for the user is that the transparent glass slide is exchanged with an opaque Si-wafer. Although they appear very different, the sample handling is practically analogous to a glass slide. The chips are quite sturdy and can easily be handled using wafer tweezers. The imaging procedure and image reconstruction is the same as in a regular dSTORM experiment. Setting up a functional chipbased microscope requires no special components, except for the photonic chips. Further details of the set-up can be found in previous work ${ }^{6,7}$. The chips used in this work have been fabricated using standard photolithography ${ }^{8}$.

Sample preparation encompasses the preparation of the sample chamber. When attaching the PDMS frame to the chip, it is crucial to avoid any small folds or rips where air might enter. If the PDMS folds when attaching it, simply remove it carefully with a tweezer and reattach it. When the sample is ready inside the PDMS chamber, the coverglass must be pressed against it, sealing the region. It is important to avoid any air bubbles 
that might form when attaching the coverglass. If an air bubble is formed, gently remove the coverglass and add PBS to the sample chamber to ensure that the sample is covered. The preparation and attachment of the cover slip can then simply be redone.

Coupling light into the waveguide is simplified using the protocol proposed in this paper. There are, however, a few common challenges that can limit coupling. Firstly, if the chip was not cleaned properly and any leftover PBS removed completely, there might be dirt or crystallized PBS on the waveguide. This can introduce major losses, resulting in very little power in the imaging region. Using a moist swab to clean the region outside the cover glass can improve the power significantly. Secondly, if the coupling facet of the waveguide is damaged (e.g., by improper handling), the coupling loss can increase drastically. Optical inspection of the edge will usually reveal any damages easily. The entire coupling facet of the chip can be polished carefully, much like an optical fiber, and will give a smooth coupling facet, which then increases the coupled power.

After the light has been coupled, the imaging procedure is the same as in any conventional dSTORM setup. If the image has inhomogeneous excitation, as demonstrated in Figure 2A, then most likely the mode averaging did not work well. The two most common reasons for this are: 1) too few images captured in order to create an average stack and 2) too short of an oscillation distance/too big of a step size. Collecting too few images can leave out some excitation patterns and the average will thus be inhomogeneous. This can easily be resolved by increasing the number of images in the average stack. Too short of an oscillation distance can also result in an inhomogeneous image, as not enough mode patterns are excited. This can also easily be resolved by increasing the oscillation distance and/or decreasing the step size. In this work we have used a piezo stage to scan the input laser beam over $20 \mu \mathrm{m}$ and acquire at least 300 images. Another approach could be to use high-speed galvo-mirrors to scan the light across the input waveguide facet within a single acquisition time, such as $10-30 \mathrm{~ms}$. This option is suitable for live cell TIRF imaging, where sub-cellular organelles are in constant motion.

Chip-based dSTORM offers an unprecedented large area TIRF excitation, which makes it ideally suited for high throughput imaging. The compact character allows for retrofitting to commercial systems, where the chip can be placed upside down for inverted setups or transparent substrates can be developed. The chips are mass fabricated and can be modified to suit many needs. Currently, the main restriction is that it is limited to 2D. The evanescent field is only available approximately $200 \mathrm{~nm}$ away from the waveguide surface, so only fluorophores within this region will be excited. Altogether, the field of integrated optics offers many opportunities for chip-based microscopy in the near future, by tackling new imaging questions as well as providing new possibilities to existing ones.

\section{Disclosures}

B.S. Ahluwalia has applied for patent GB1606268.9 for chip-based optical nanoscopy. The other authors declare no competing financial interests.

\section{Acknowledgments}

The authors would like to acknowledge The European Research Council (grant no. 336716 to B.S.A.). The authors would also like to thank Irati Lagfragua for her invaluable assistance with recording and editing the video.

\section{References}

1. Juette, M.F. et al. Three-dimensional sub-100 nm resolution fluorescence microscopy of thick samples. Nature Methods. 5 (6), $527-529$ (2008).

2. Huang, B., Wang, W., Bates, M., Zhuang, X. Three-Dimensional Super-Resolution Imaging by Stochastic Optical Reconstruction Microscopy. Science. 319 (5864), 810-813 (2008).

3. Lampe, A., Haucke, V., Sigrist, S.J., Heilemann, M., Schmoranzer, J. Multi-colour direct STORM with red emitting carbocyanines. Biology of the Cell. 104 (4), 229-237 (2012).

4. Wazawa, T., Ueda, M. Total internal reflection fluorescence microscopy in single molecule nanobioscience. Advances in Biochemical Engineering/Biotechnology. 95, 77-106 (2005).

5. Jalali, B., Fathpour, S. Silicon photonics. Journal of Lightwave Technology. 24 (12), 4600-4615 (2006).

6. Helle, Ø.I., Coucheron, D.A., Tinguely, J.-C., Øie, C.I., Ahluwalia, B.S. Nanoscopy on-a-chip: super-resolution imaging on the millimeter scale. Optics Express. 27 (5), 6700-6710 (2019).

7. Diekmann, R. et al. Chip-based wide field-of-view nanoscopy. Nature Photonics. 11 (5), 322-328 (2017).

8. Tinguely, J.-C., Helle, Ø.I., Ahluwalia, B.S. Silicon nitride waveguide platform for fluorescence microscopy of living cells. Optics Express. 25 (22), 27678-27690 (2017).

9. Ahluwalia, B.S., McCourt, P., Huser, T., Hellesø, O.G. Optical trapping and propulsion of red blood cells on waveguide surfaces. Optics Express. 18 (20), 21053-21061 (2010).

10. Coucheron, D.A., Wadduwage, D.N., Murugan, G.S., So, P.T.C., Ahluwalia, B.S. Chip-Based Resonance Raman Spectroscopy Using Tantalum Pentoxide Waveguides. IEEE Photonics Technology Letters. 31 (14), 1127-1130 (2019).

11. Helle, Ø.I., Dullo, F.T., Lahrberg, M., Tinguely, J.-C., Ahluwalia, B.S. Structured illumination microscopy using a photonic chip. ArXiV. https:// arxiv.org/abs/1903.05512v1 (2019).

12. Metcalf, D.J., Edwards, R., Kumarswami, N., Knight, A.E. Test Samples for Optimizing STORM Super-Resolution Microscopy. Journal of Visualized Experiments. (79) (2013).

13. Ovesný, M., Křižek, P., Borkovec, J., Švindrych, Z., Hagen, G.M. ThunderSTORM: a comprehensive ImageJ plug-in for PALM and STORM data analysis and super-resolution imaging. Bioinformatics. 30 (16), 2389-2390 (2014).

14. Archetti, A., Glushkov, E., Sieben, C., Stroganov, A., Radenovic, A., Manley, S. Waveguide-PAINT offers an open platform for large field-ofview super-resolution imaging. Nature Communications. 10 (2019). 
15. Braet, F., Wisse, E. Structural and functional aspects of liver sinusoidal endothelial cell fenestrae: a review. Comparative Hepatology. 1, 1 (2002). 